\title{
Chest CT Findings and Differential Diagnosis of Mycoplasma pneumoniae Pneumonia and Mycoplasma pneumoniae Combined with Streptococcal Pneumonia in Children
}

\author{
Jing Wang $\left(\mathbb{D},{ }^{1}\right.$ Chen Xia $\mathbb{D}^{1},{ }^{1}$ Ashutosh Sharma $\mathbb{D}^{\circ},{ }^{2}$ Gurjot Singh Gaba ${ }^{\circ},{ }^{3}$ \\ and Mohammad Shabaz $\left.{ }^{4}\right)^{4}$ \\ ${ }^{1}$ Department of Infectious Diseases, Children's Hospital of Nanjing Medical University, Nanjing, Jiangsu 210008, China \\ ${ }^{2}$ Institute of Computer Technology and Information Security, Southern Federal University, Rostov-on-Don, Russia \\ ${ }^{3}$ School of Electronics and Electrical Engineering, Lovely Professional University, Phagwara 144411, India \\ ${ }^{4}$ Arba Minch University, Arba Minch, Ethiopia
}

Correspondence should be addressed to Chen Xia; xiachen1020@outlook.com and Mohammad Shabaz; mohammad.shabaz@ amu.edu.et

Received 7 April 2021; Revised 22 May 2021; Accepted 2 June 2021; Published 15 June 2021

Academic Editor: Anupam Kumar Bairagi

Copyright $\odot 2021$ Jing Wang et al. This is an open access article distributed under the Creative Commons Attribution License, which permits unrestricted use, distribution, and reproduction in any medium, provided the original work is properly cited.

Background. In this day and age, $17 \%$ of children less than 5 years of age died of pneumonia; it is the common cause of children death. It is one of the main children respiratory infectious diseases, i.e., mycoplasma pneumonia (MP). The imaging examination can be adopted to quickly observe the morphology and scope of the pulmonary lesions and know the effect of disease treatment and subsequent changes in the disease in order to provide a basis for treatment. Therefore, the most commonly applied technology for detecting pneumonia in children is imaging technology, including chest X-ray and CT. Objectives. The main objective of the work is to investigate the chest computed tomography (CT) findings of children patients with Mycoplasma pneumoniae pneumonia (MPP) and MP combined with streptococcal pneumonia (SP). The mixed infection of MP and SP is very common clinically, and the diagnosis of this type of mixed pneumonia is a critical research topic faced by pediatric respiratory physicians. The comparison is done on the incidence of bronchial and pulmonary interstitial lesions, the degree of lymph node enlargement, the volume and depth of pleural effusion, and the location and morphology of the pulmonary lesions in the chest CT images of children patients from the two groups. Methods. There were comparisons on the incidence of bronchial and pulmonary interstitial lesions, the degree of lymph node enlargement, the volume and depth of pleural effusion, and the location and morphology of the pulmonary lesions in the chest CT images of children patients from the two groups. All the experiments are done in the MATLAB. Results. The results showed that the proportions of reticular shadow, ground glass shadow, bronchial inflation phase, tube wall thickening, and vascular bundle thickening on the CT images of children patients from the MPP group were dramatically higher than those of the MP + SP group $(P<0.05)$. The maximum transverse diameter of enlarged lymph node in children patients from the MPP group was obviously larger than the diameter of the MP + SP group $(P<0.05)$. The number of children patients with pleural effusion was 22 in the MP + SP group, which was greatly higher than the MPP group $(P<0.05)$. Conclusion. In conclusion, the chest CT images of children patients from the MPP group were mainly pulmonary interstitial changes. Furthermore, the alveolar inflammation could be observed on the CT images shown when children patients were combined with SP infection. The more obvious manifestations were that the flaky shadows appeared in the lungs, the pleural effusion became thicker, and the transverse diameters of enlarged lymph nodes were bigger. 


\section{Introduction}

Childhood pneumonia is the main cause of death for children under the age of 5 years, and SP is the most common type of pneumonia from the $20^{\text {th }}$ day of birth to the entire childhood. The MP infection rate in children that are older than 5 years old is more than 50\% [1]. The mixed infection of MP and SP is very common clinically, and the diagnosis of this type of mixed pneumonia is a critical research topic faced by pediatric respiratory physicians. Accurate imaging diagnosis can help clinicians to treat the disease in time and use drugs rationally, so as to avoid delay in the conditions of patients.

Nowadays, $17 \%$ of children less than 5 years of age died of pneumonia; it is the common cause of children death $[2,3]$. It is one of the main children respiratory infectious diseases, that is, mycoplasma pneumonia. In the initial age, there may or may not be clinical symptoms which cause the lesions outside the respiratory system. Several days are required for the completion of the serologic diagnosis for making decision of initial medication which is critical for the community-acquired pneumonia treatment [3]. The images for childhood pneumonia and the fight against pneumonia are shown in Figure 1.

Pneumonia is the most frequent disease and its main causes are pathogen infection and body allergic reactions. In the past 3 years, MPP has become a common pneumonia in children and adolescents, accounting for $10 \%-40 \%$ of community-acquired pneumonia, and its infection rate is $10 \%-20 \%[4,5]$. Compared with simple MP, combined SP is more likely to cause severe pneumonia. It can damage the pulmonary tissue and seriously endanger the health of children. Clinical studies have confirmed that mixed-infected children with pneumonia have a longer course and are more likely to develop pulmonary lesions and pleural effusion. Among them, SP is the most common [6] and it is gradually being paid attention to in clinic. Thus, effective identification of MP infection and mixed infection of MP and streptococcus has vital reference significance for the treatment of childhood pneumonia. The imaging examination can be adopted to quickly observe the morphology and scope of the pulmonary lesions and know the effect of disease treatment and subsequent changes in the disease in order to provide a basis for treatment. Therefore, the most commonly applied technology for detecting pneumonia in children is imaging technology, including chest X-ray and CT.

The multislice spiral CT method is used with a scanning layer, and there are different conditions for scanning. Regular chest CT scans of children are done in calm breathing state. The underwent breathless scanning is held by the older children. The MP antibody is detected by the passive agglutination method (MP-IgM) with patient's serum twice every 10 or 14 days [7]. The two experienced radiologists assessed the $\mathrm{CT}$ imaging and retrospectively diagnosed the diseases including the lesion characteristics and lesions involving lobes. The ground glass opacity (GGO), the consolidation opacity, and the mass opacity are included by the lesion types. Peribronchovascular nodules are presented in the bronchovascular bundle, and mildly increased attenuation without obscuration of the underlying vasculature is known as GGO $[8,9]$. There are three types of nodules:

(1) Centrilobular nodules in the centrilobular location

(2) Peribronchovascular nodules in the bronchovascular bundles

(3) Granulomas

Studies have shown that interstitial changes are the pathological basis of MPP [10]. It is the first to damage the body's terminal and respiratory bronchiolar epithelial cells, so as to cause the parabronchial tissues to infiltrate into the interlobular septum, thereby resulting in thickening of the interlobular septum and edema of the bronchiole wall. The lesions are mainly concentrated in the small and medium airways [11]. The cuffing signs and thickening of the bronchial wall can be observed in the thin-layer CT images of the chest, showing ring sign, orbit sign, and thickening of the bronchial vascular bundle [12]. If the condition is mild, the interlobular space will be thickened, and a small part of the pulmonary tissues will be also thickened. However, most will become asymmetric and irregular high-density small shadows. At this time, the lesions will merge with each other. The invasion function of bacterial capsule is the main therapeutic factor of SP. Invasion of tissues first causes edema of the alveolar wall, and then, white blood cells and red blood cells exudate. At this time, the alveoli are quickly and directly invaded by bacteria. The bacteria can even invade directly, causing symptoms such as congestion and edema in the pulmonary lobes.

Many researchers have worked on differential diagnosis of Mycoplasma pneumoniae pneumonia and Mycoplasma pneumoniae combined with streptococcal pneumonia in children for many years. The scientific basis for clinical diagnosis and severity assessment are provided for the improvement of comprehension of the imaging findings for the children chest imaging discussion. In the pediatric department, 126 cases of children are analyzed [13]. With chest computed tomography, this paper aims to clarify the abnormalities pattern with Mycoplasma pneumoniae pneumonia, and $M$. pneumoniae pneumonia from Streptococcus pneumoniae pneumonia is distinguished through the radiographic findings [14]. The CT case of different cases is performed by the retrospective review. The bilateral bronchial wall thickening or centrilobular nodules were seen in the patients. In the same patients, CT findings between early stage and progressed stage are compared and in the progressed stage, early stage radiographic features were not clearly observed. The wall thickening and centrilobular nodules in the CT outcomes are found by the M. pneumoniae pneumonia diagnosis. From the recent publications, the comprehensive radiological literature review is provided on ongoing radiological investigation of the imaging features of the chest ultrasound (US), radiographs (CXR), and computed tomography (CT) examinations. The evaluation and analysis of multimodality 


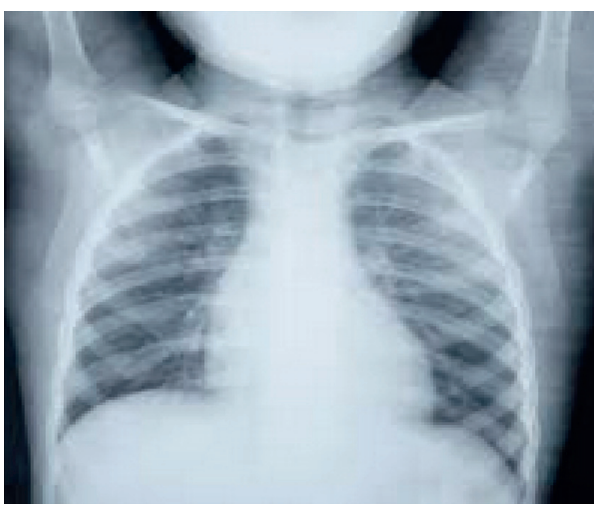

(a)

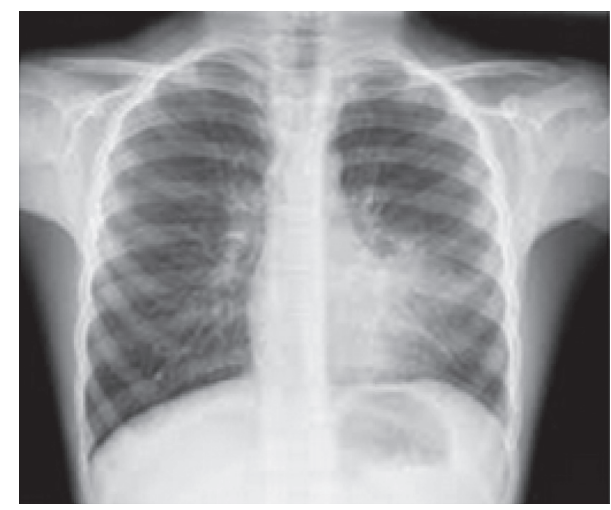

(b)

Figure 1: (a) Childhood pneumonia. (b) Fight against pneumonia.

imaging outcomes are done [15]. In many patients, the different tests like chest US, CXR, and CT were performed. The glenohumeral instability of patient's MRI is analyzed, and torn labrum diagnosis is determined which can be confirmed by the surgical exploration [16]. The correct labral tear diagnoses are $80 \%$ obtained after the analysis. A clinical, radiological, and histological entity is organizing pneumonia which is classified as interstitial lung disease [17]. The new understandings of the clinical and histological presentation are summarized in this work, and the most relevant CT features are reviewed. The author in this paper details many complications like pneumonia commonly accompanied by emphysema. The usual disease progression is changed by the destroyed airspaces [18]. Various cases of common comorbidities are demonstrated by the authors with unusual radiographic findings in emphysema patients. The proper management of emphysema patients is commonly diagnosed by the various emphysema findings. In this paper, the author details the radiographic findings which are nonspecific in mycoplasma pneumonia [19]. A single lobe is confined by the focal reticulonodular opacification which is radiographic pattern and closely associated with the mycoplasma infection. The mycoplasma pneumonia diagnosis is considered when bilateral reticulonodular opacification is seen. Due to confluent interstitial shadows, transient pseudoconsolidations are seen often. For the mycoplasma pneumonia diagnosis, radiographic findings are not sufficient. By combining the clinical findings, diagnosis accuracy is increased. The author describes the radiological features in adult patients with $\mathrm{H} 1 \mathrm{~N} 1$ influenza pneumonia [20]. During the epidemic of H1N1 influenza infection, descriptive study of retrospective data is performed. By RTPCR, H1N1 influenza virus infection is confirmed by the 209 adult patients. The opacities were mainly bilateral, basal, and midzonal in CXR and peripheral in CT. In the $\mathrm{H} 1 \mathrm{~N} 1$ pneumonia, the predominant radiological pattern is bilateral and alveolar consolidation. To maximise iodine detection, preprocessing is done by the author in this paper for chest imaging in acute and chronic embolic diseases demonstration [21]. The work aims to set out the physical basis for the technology and postprocessing protocols used and to present future developments.

The literature lacks the investigation of chest computed tomography (CT) findings of children patients with $M y$ coplasma pneumoniae pneumonia (MPP) and MP combined with streptococcal pneumonia (SP), which is very important. For the differential diagnosis references and treatment of MPP in clinic, reference is required. Clinically, the MP and SP mixed infection is very common, and the mixed pneumonia diagnosis is a critical research topic faced by pediatric respiratory physicians. In this paper, the main contribution of the work is to investigate the chest computed tomography (CT) findings of children patients with Mycoplasma pneumoniae pneumonia (MPP) and MP combined with streptococcal pneumonia (SP). In this study, 80 children patients with pneumonia were selected and were admitted to the Department of Respiratory Medicine of Indira Gandhi Medical Hospital from June 2019 to June 2020. Besides, 40 cases with MPP and 40 cases with MP combined with SP were enrolled into a MPP group and a MP + SP group in turn based on the results of serum MP-IgM detection and streptococcal blood culture. There were comparisons on the incidence of bronchial and pulmonary interstitial lesions, the degree of lymph node enlargement, the volume and depth of pleural effusion, and the location and morphology of the pulmonary lesions in the chest CT images of children patients from the two groups.

\section{Materials and Methods}

2.1. Clinical Research Objects. From June 2019 to June 2020, 80 children patients admitted to the Department of Respiratory Medicine of Indira Gandhi Medical Hospital for one year were selected as the research objects, who suffered from MMP and MP combined with SP confirmed by blood culture and serological examinations in turn. Among them, there were 40 cases in the MPP group, including 19 boys and 21 girls. Besides, they were 3-12 years old, with an average age of $7.50 \pm 2.66$ years [22-24]. There were also 40 cases in the $\mathrm{MP}+\mathrm{SP}$ group ( 22 boys and 18 girls), with the age of $5-14$ years (average age of $8.78+2.99$ years). The differences 
in gender and age of the two groups were not statistically obvious $(P>0.05)$. All children patients underwent chest CT detections before receiving antibiotic treatment.

2.2. Operating Equipment and Methods. The children patients from the MPP group and the MP + SP group were given with chest CT scanning before treatment. The multislice spiral CT machine was adopted in this experiment [25]. What's more, they were asked to maintain breathholding during the examination. The scanning range included chest cavity to lung base, with the adjustment of related parameters, and the corresponding data were uploaded to the workstation after scanning.

2.3. Observation Indicators. The 3 experienced imaging doctors examined all the research objects in this study and observed the CT images together in terms of the signs of bronchial and pulmonary interstitial lesions, the transverse diameter of lymph nodes and other specific manifestations, and the incidence and depth of pleural effusion. Then, the location and morphology of lesions in children patients from the two groups were analyzed based on the above observation indicators. According to these symptoms in children with pneumonia, the corresponding lesions might be judged [26, 27]. The lesion distribution presented on the CT images could be classified into consolidation, ground glass shadow, and reticular shadow on the basis of imaging density and divided according to lung lobes (anatomical unit) into left and right sides, and upper and lower lobes.

2.4. Statistical Methods. The measurement data were represented by mean \pm standard deviation $(\bar{x} \pm s)$, and the disordered classification data were expressed as percentage (\%). SPSS 20.0 software was employed to analyze the difference between the two groups [28]. Above all, $P>0.05$ meant that the difference was not statistically substantial, and $P<0.05$ indicated there was a statistically marked difference.

2.5. Clinical Features of Mycoplasma Infection. For mycoplasma pneumonia, humans are the only known reservoir. It is the tiny organism which is less than $350 \mu \mathrm{m}$ and not visible at light microscopy [5]. This infection is spread by direct contact for the period of 1-2 weeks. It is an important cause of community-acquired respiratory infections in school-age children. Mycoplasma pneumonia illness is gradual, and its symptoms are nonspecific. The illness may begin in the upper respiratory tract and is often accompanied by lowgrade fever, headache, and myalgias [29-32]. The rales, rhonchi, and decreased breath sounds are the different clinical signs included in the lungs mycoplasma infection. Usually the white blood cell count is in normal range. The respiratory symptoms precede the nonrespiratory manifestations in most of the cases, but there is little effect of pulmonary disease on subsequent neurologic disease [33].

\section{Results and Discussion}

3.1. Children with Bronchial and Interstitial Lung Disease from the Two Groups. The chest CT findings of bronchial and pulmonary interstitial lesions in children patients from the MPP group and MP + SP group were observed and compared, and the comparison results are shown in Table 1 and graphically shown in Figure 2. The CT images of children patients from the MPP group showed reticular shadows, ground glass shadows, reticular shadows, bronchial inflation phase, tube wall thickening, and vascular bundle thickening, and the appearance proportion of the above in the MPP group was higher obviously than that of the $\mathrm{MP}+\mathrm{SP}$ group $(P=0.03$ and $P<0.05)$ [34]. Besides, Figure 3 indicates the specific signs of chest CT in one child patient from the MPP group (the image of this patient was typical). The proportions of cases with bronchopulmonary emphysema and bronchial inflation phase from the two groups were compared, with no statistically obvious difference $(P=0.94$ and $P>0.05)$.

\subsection{Children with Pleural Effusion and Lymph Node En-} largement from the Two Groups. The largest transverse diameter of enlarged lymph nodes in the MPP group and the $\mathrm{MP}+\mathrm{SP}$ group was $7.23 \pm 2.38 \mathrm{~mm}$ and $9.68 \pm 2.95 \mathrm{~mm}$, respectively. It was found that the transverse diameter of enlarged lymph nodes in children patients from the MP + SP group was greatly larger than that of the MPP group $(P=0.04$ and $P<0.05$ ) (Table 2). The depth of pleural effusion in children patients from the MPP group was $3.35 \pm 2.23 \mathrm{~mm}$, while the depth of the MP + SP group was $12.75 \pm 11.36 \mathrm{~mm}$ $[35,36]$. Thus, the depth of pleural effusion in children patients from the MP + SP group rose hugely in contrast to the depth of the MPP group $(P=0.009$ and $P<0.05)$. Figure 4 shows that the incidence of pleural effusion in children patients from the MPP group (22\%) reduced steeply in contrast to the incidence of the $\mathrm{MP}+\mathrm{SP}$ group (55\%) $(P=0.031$ and $P<0.05)$.

\subsection{The Pathogenic Sites in the Lungs of the Sick Children.} There was a comparison of the pathogenic sites of children patients from the MPP group and MP + SP group $(P>0.05)$, and the results are presented in Tables 3 and 4 and graphically shown in Figures 5 and 6 for better analysis and visualization.

3.4. Morphology of Pulmonary Lesions in Sick Children. In the MPP group, 33 cases had fan-shaped thin slice shadows in the lungs, and the incidence was $83 \%$. Besides, there were 7 cases with fan-shaped thin slice shadows in the $\mathrm{MP}+\mathrm{SP}$ group (18\%). Thus, the above was statistically different $(P<0.05)$. As for irregular pulmonary consolidation, there were 36 in the MP + SP group (90\%) and 9 in the MPP group (23\%), and the difference was statistically substantial $(P<0.05)$ (Figure 7$)$.

The results of this study revealed that the proportion of reticular shadow, ground glass shadow, bronchial inflation phase, tube wall thickening, and vascular bundle thickening in chest CT images of the MPP group was markedly higher than that of the MP + SP group. The damage of MPP was 
TABLE 1: Chest CT findings and infection rate of bronchial and pulmonary interstitial lesions in children patients from the MPP group and $\mathrm{MP}+\mathrm{SP}$ group (\%).

\begin{tabular}{lcccc}
\hline \multirow{2}{*}{ Chest CT findings } & \multicolumn{2}{c}{ MPP group $(n=40)$} & \multicolumn{2}{c}{ MP+SP group $(n=40)$} \\
& Number of cases & Infection rate (\%) & Number of cases & Infection rate (\%) \\
\hline Bronchopulmonary emphysema & 8 & 15 & 10 & 25 \\
Reticular shadow & 34 & 80 & 72 & 30 \\
Ground glass shadow & 24 & 60 & 32 & 18 \\
Bronchial inflation phase & 37 & 93 & 20 & 50 \\
Tube wall thickening & 38 & 95 & 13 & 33 \\
Vascular bundle thickening & 32 & 70 & & 70 \\
\hline
\end{tabular}

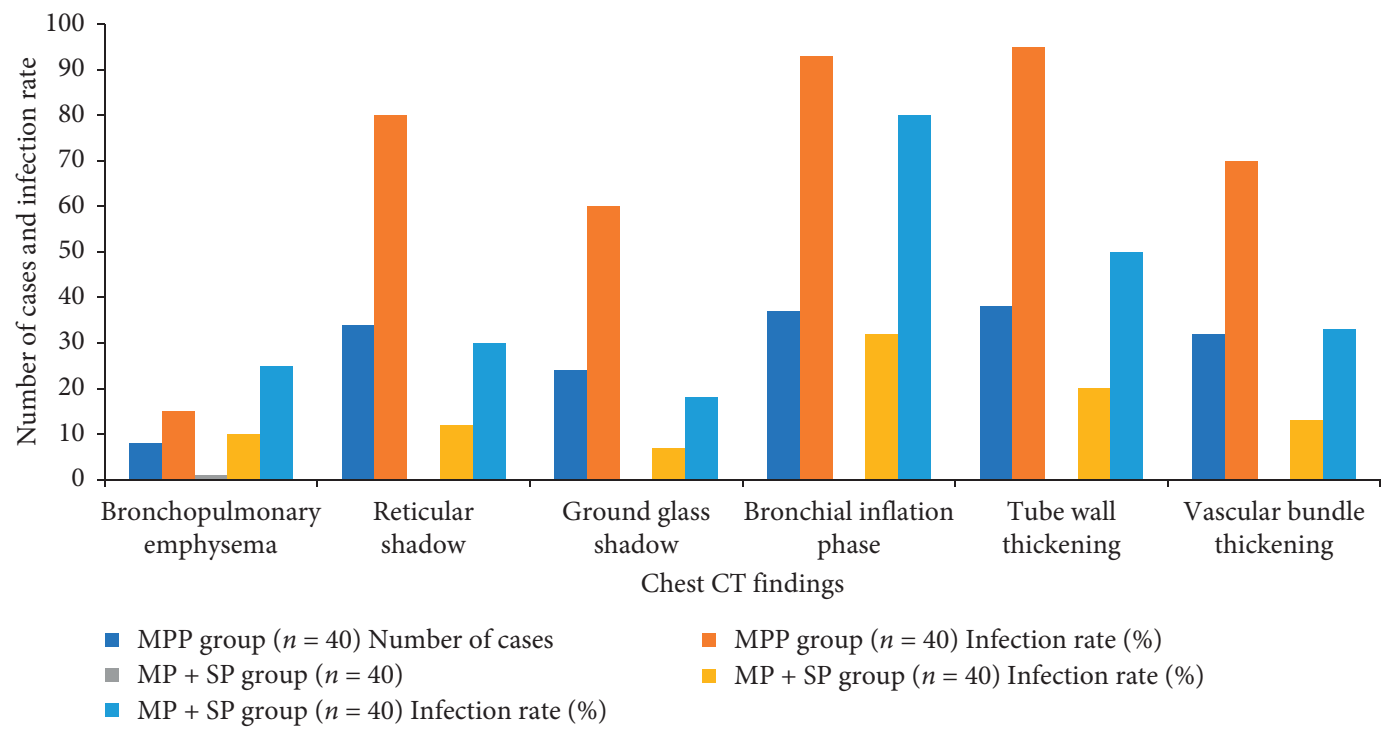

Figure 2: Chest CT findings and infection rate of bronchial and pulmonary interstitial lesions.

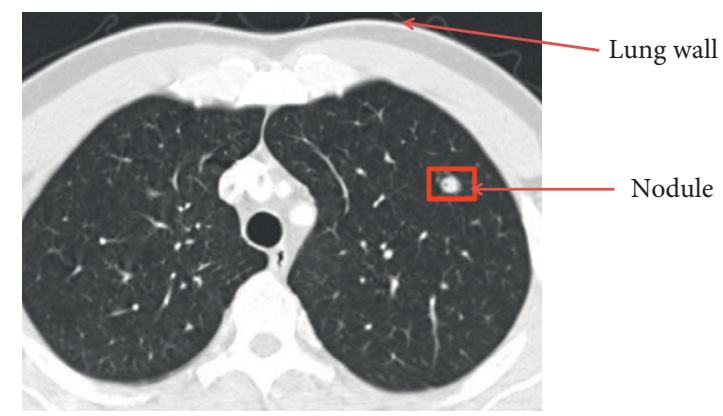

FIgUre 3: Chest CT image of one child patient from the MPP group.

TABLE 2: Comparison of the depth of pleural effusion and the maximum transverse diameter of lymph nodes in children patients from both groups $(\bar{x} \pm s)$.

\begin{tabular}{lcc}
\hline Group & $\begin{array}{c}\text { Depth of } \\
\text { pleural effusion }\end{array}$ & $\begin{array}{c}\text { Maximum transverse } \\
\text { diameter of lymph nodes }\end{array}$ \\
\hline MPP group $(n=40)$ & $3.35 \pm 2.23$ & $7.23 \pm 2.38$ \\
MP + SP group $(n=40)$ & $12.75 \pm 11.36$ & $9.68 \pm 2.95$ \\
$P$ value & $<0.05$ & $<0.05$ \\
\hline
\end{tabular}




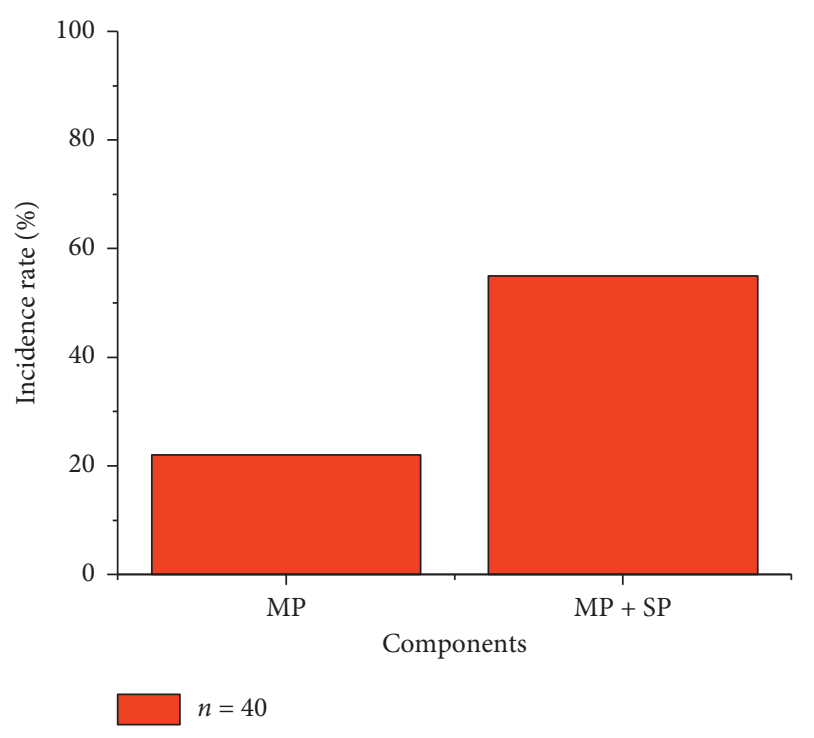

Figure 4: The incidence of pleural effusion in children patients from the two groups.

TABle 3: Comparison of the left lung lesions of children patients from the two groups.

\begin{tabular}{|c|c|c|c|c|c|}
\hline \multirow{3}{*}{ Group } & \multirow{3}{*}{ Number of cases } & \multicolumn{4}{|c|}{ Left lung } \\
\hline & & \multicolumn{2}{|c|}{ Upper lobe } & \multicolumn{2}{|c|}{ Lower lobe } \\
\hline & & Number of cases & $\%$ & Number of cases & $\%$ \\
\hline MPP group & 40 & 20 & 50 & 14 & 35 \\
\hline $\mathrm{MP}+\mathrm{SP}$ group & 40 & 15 & 38 & 17 & 43 \\
\hline$P$ value & & & & & \\
\hline
\end{tabular}

TABLE 4: Comparison of the right lung lesion sites of children patients from the two groups.

\begin{tabular}{|c|c|c|c|c|c|c|c|}
\hline \multirow{3}{*}{ Group } & \multirow{3}{*}{ Number of cases } & \multicolumn{6}{|c|}{ Right lung } \\
\hline & & \multicolumn{2}{|c|}{ Upper lobe } & \multicolumn{2}{|c|}{ Middle lobe } & \multicolumn{2}{|l|}{ Upper lobe } \\
\hline & & Number of cases & $\%$ & Number of cases & $\%$ & Number of cases & $\%$ \\
\hline MPP group & 40 & 10 & 25 & 14 & 35 & 11 & 28 \\
\hline $\mathrm{MP}+\mathrm{SP}$ group & 40 & 13 & 33 & 12 & 30 & 15 & 38 \\
\hline$P$ value & & & & $>0.05$ & & & \\
\hline
\end{tabular}

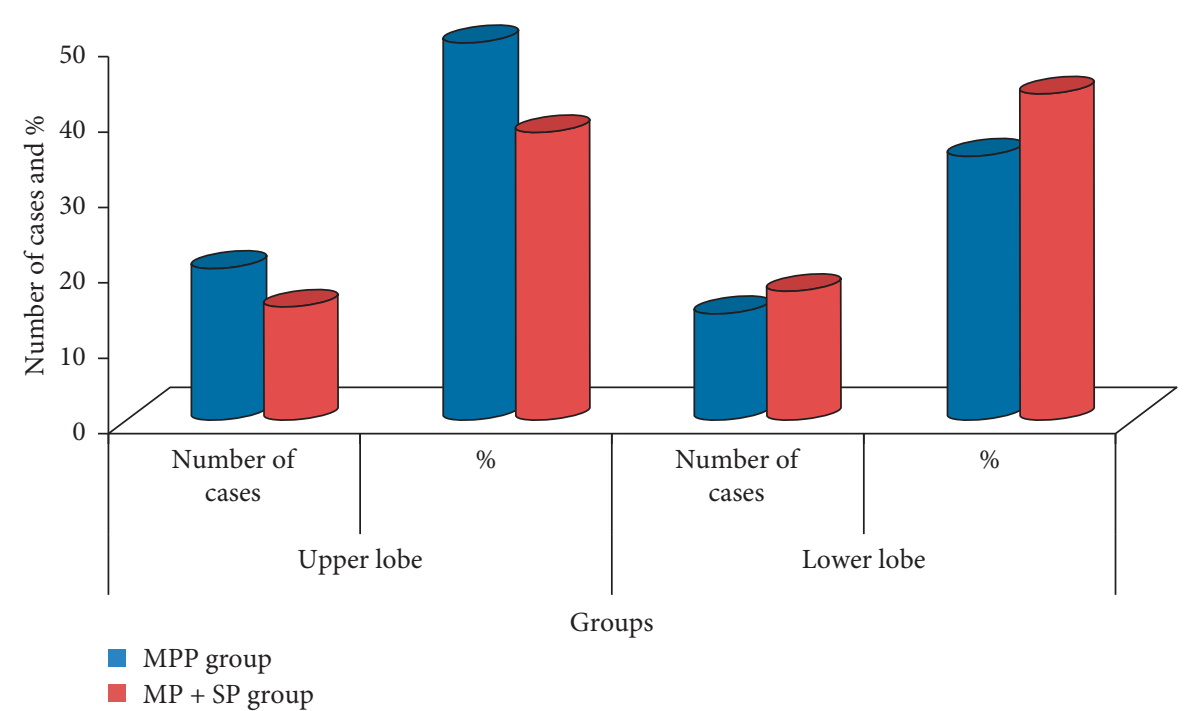

FIgURE 5: Left lung lesions of children patients from the two groups. 


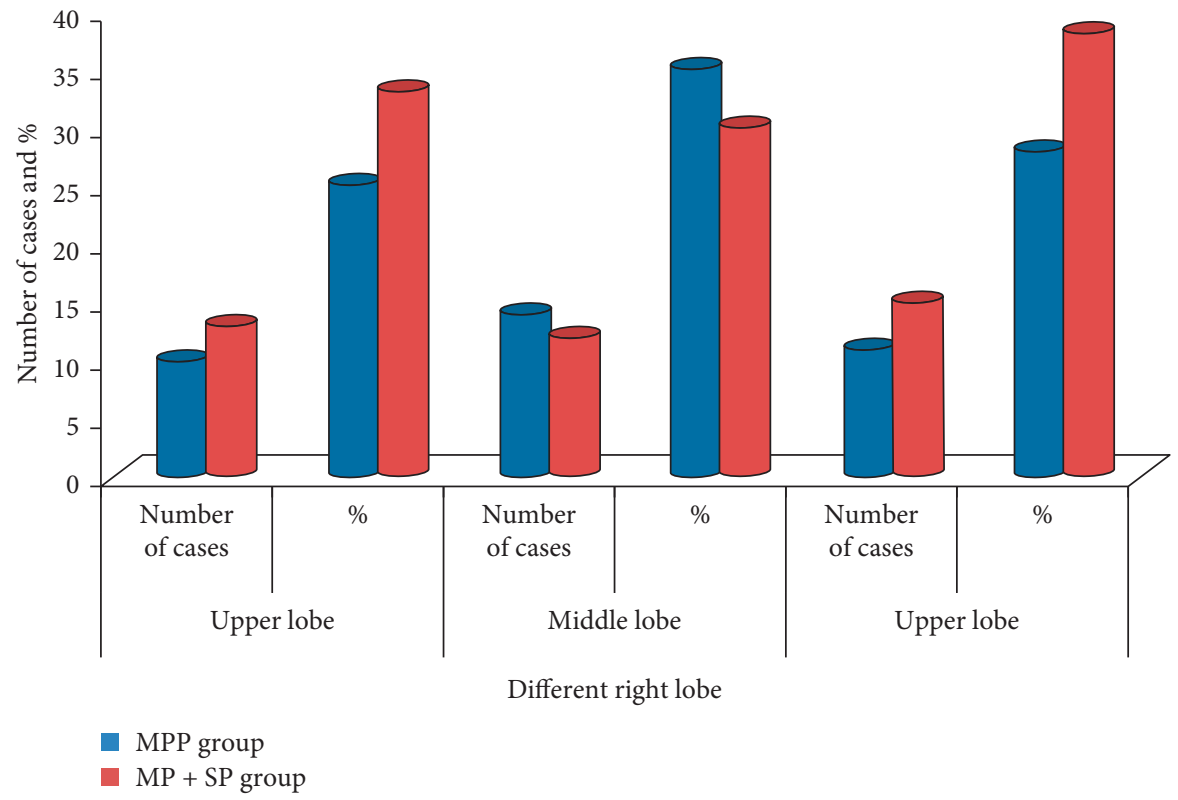

FIGURE 6: Right lung lesion sites of children patients from the two groups.

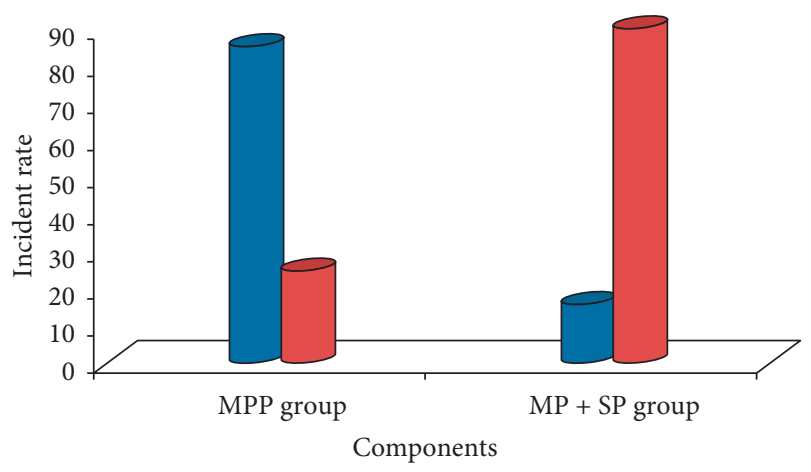

- Fan shaped shadow

- Irregular pulmonary consolidation shadows

Figure 7: The incidence of two types of pulmonary lesions in children patients from the two groups.

most representative in the terminal and respiratory bronchiole epithelium, so the accumulation and infiltration of mononuclear lymphocytes will occur in some tissues clinically, and eventually the symptom of bronchopulmonary emphysema will appear. Therefore, thickening of the bronchial wall and the bronchial vascular bundle could be discovered on the chest CT images. With the progression of the disease, the surrounding tissues of the bronchiole would be infiltrated by inflammation again, so that the interlobular septa were thickened [37-39]. The alveolar cavity was the main site of SP inflammation, leading to the symptoms of pulmonary congestion and edema.

\section{Discussion}

The inflammatory response of MP combined with SP is more severe than that of MPP alone, and it is manifested as a more substantial local inflammatory exudation rate and less air bubbles in the alveolar cavity. On the CT images, the translucency of the lung field was reduced [40-42]. However, a large area of dense shadows in the lungs could be observed on the CT images when a large amount of inflammatory exudation led to the loss of air in the alveoli, which could block the pulmonary interstitial changes caused by MPP [43]. Therefore, the proportion of ground glass shadow and reticular shadow in children patients from the MP + SP group decreased sharply. Studies have pointed out that hilar lymph node enlargement has occurred in about $1 / 5$ of patients with pneumonia, most of which have been unilateral. Lymph node enlargement behind the anterior tracheal vena cava was observed in all children patients in this study, which was basically the same as the research results of relevant literature. In addition, the maximum transverse diameter of lymph node enlargement in children patients from the MPP group $(7.23 \pm 2.38 \mathrm{~mm})$ dropped hugely compared with the $\mathrm{MP}+\mathrm{SP}$ group $(9.68 \pm 2.95 \mathrm{~mm})$. It indicated that children patients with obvious lymph node enlargement in the actual clinical setting had the possibility of mixed infection; for example, the maximum transverse diameter was more than $0.9 \mathrm{~cm}$.

In this study, the incidence of pleural effusion in children patients from the MPP group was $22 \%$, while the incidence of pleural effusion in the MP + SP group was 55\%. Thus, the above incidence of the MP $+\mathrm{SP}$ group was dramatically higher than that of the MPP group, which was mainly caused by the inflammatory exudation after MP infection and the induced pleural response [44-46]. Inflammation can further enhance the permeability of the local capillary wall. At this time, the inflammatory exudate increases, thus making the pleural effusion obviously more. For all the children patients with pneumonia with relatively more pleural effusion, the possibility of suffering from MP combined with SP could not be excluded. 
From the perspective of the pathogenesis of MPP and SP, there is little difference in the sites of the two types of pneumonia. It was consistent with the research of left and right pulmonary lesions in this study. Left lung lesions were slightly greater than right lung lesions, but there was no difference between the two types of pneumonia.

In this study, the irregular pulmonary consolidation shadows appeared in the CT images of 36 children patients from the MP + SP group, with an incidence of $90 \%$, which was markedly higher than the incidence of the MPP group. There were consolidation shadows with irregular shapes after the onset of lesions, and this was because of the result of lesion fusion due to the inflammation of alveoli. It was also in line with the pathologic findings of MP combined with SP.

\section{Conclusion}

Pneumonia is the most frequent disease in children, and its main causes are pathogen infection and body allergic reactions. In the past 3 years, MPP has become a common pneumonia in children and adolescents, accounting for $10 \%-40 \%$ of community-acquired pneumonia, and its infection rate is $10 \%-$ $20 \%$. For the sake of medical ethics, the differences in the two types of pneumonia were observed on the basis of CT detection. However, there is a lack of comparison of the chest X-ray examinations of children patients from the two groups. The 3 experienced imaging doctors examined all the research objects in this study and observed the CT images together in terms of the signs of bronchial and pulmonary interstitial lesions, the transverse diameter of lymph nodes and other specific manifestations, and the incidence and depth of pleural effusion. Then, the location and morphology of lesions in children patients from the two groups were analyzed based on the above observation indicators. It is planned to carry out further comparison of the differential diagnosis between the two groups by chest X-ray examination, which may be more convincing and is better to expand the application of chest X-ray in the treatment of MPP and SP. The irregular pulmonary consolidation shadows appeared in the CT images of 36 children patients from the MP + SP group, with an incidence of $90 \%$, which was markedly higher than the incidence of the MPP group; the alveolar inflammation could be observed on the CT images shown when children patients were combined with SP infection. The more obvious manifestations were that the flaky shadows appeared in the lungs, the pleural effusion became thicker, and the transverse diameters of enlarged lymph nodes were bigger. There were consolidation shadows with irregular shapes after the onset of lesions, and this was because of the result of lesion fusion due to the inflammation of alveoli. It was also in line with the pathologic findings of MP combined with SP. The investigation of the emerging COVID-19 pneumonia's imaging features on chest ultrasound (US) and computed tomography (CT) examinations can be focused on in the future research.

\section{Data Availability}

The data are available upon request to the corresponding author.

\section{Conflicts of Interest}

The authors have no conflicts of interest.

\section{Acknowledgments}

The authors acknowledge Indira Gandhi Medical Hospital for providing data.

\section{References}

[1] W. S. Chen, H. H. Zhu, and G.-S. Feng, "Treating leukemia at the risk of inducing severe anemia," Experimental Hematology, vol. 44, no. 5, pp. 329-331, 2016.

[2] M. Ohana, M. Y. Jeung, A. Labani, S. El Ghannudi, and C. Roy, "Thoracic dual energy CT: acquisition protocols, current applications and future developments; scanographie double energie en pathologie thoracique: protocoles d'exploration, applications actuelles et developpements," Journal de Radiologie Diagnostique et Interventionnelle, vol. 95, 2014.

[3] J. K. Mansel, E. C. Rosenow, T. F. Smith, and J. W. Martin, "Mycoplasma pneumoniae pneumonia," Chest, vol. 95, no. 3, pp. 639-646, 1989.

[4] C. Kang, Y. Vali, M. Naeem, and R. Reddy, "Cryptogenic organising pneumonia presenting with spontaneous pneumothorax and the value of procalcitonin: a case report," Respiratory Medicine Case Reports, vol. 22, pp. 36-38, 2017.

[5] H. F. Zhang, H. T. Bai, J. M. Li, H. Xie, and Y. Wang, "Association of drug resistance of Mycoplasma pneumoniae with DNA load and genotypes in children with Mycoplasma pneumoniae pneumonia," Zhongguo Dang Dai Er Ke Za Zhi, vol. 19, no. 11, pp. 1180-1184, 2017.

[6] C. H. Park, E. V. Valore, A. J. Waring, and T. Ganz, "Hepcidin, a urinary antimicrobial peptide synthesized in the liver," Journal of Biological Chemistry, vol. 276, no. 11, pp. 7806-7810, 2001.

[7] Y. S. Youn, K. Y. Lee, J. Y. Hwang et al., "Difference of clinical features in childhood Mycoplasma pneumoniae pneumonia," BMC Pediatrics, vol. 10, no. 1, pp. 48-57, 2010.

[8] Y.-S. Youn and K.-Y. Lee, "Mycoplasma pneumoniaepneumonia in children," Korean Journal of Pediatrics, vol. 55, no. 2, p. 42, 2012.

[9] A. Sharma and R. Kumar, "Computation of the reliable and quickest data path for healthcare services by using servicelevel agreements and energy constraints," Arabian Journal for Science and Engineering, vol. 44, no. 11, pp. 9087-9104, 2019.

[10] M. J. Søndergaard, M. B. Friis, D. S. Hansen, and I. M. Jørgensen, "Clinical manifestations in infants and children with Mycoplasma pneumoniae infection," PLoS One, vol. 13, no. 4, 2018.

[11] T. Saraya, T. Watanabe, Y. Tsukahara et al., "The correlation between chest $\mathrm{X}$-ray scores and the clinical findings in children and adults with mycoplasma pneumoniae pneumonia," Internal Medicine, vol. 56, no. 21, pp. 2845-2849, 2017.

[12] Y.-l. Meng, W.-m. Wang, D.-d. Lv et al., "The effect of Platycodin D on the expression of cytoadherence proteins P1 and P30 in Mycoplasma pneumoniae models," Environmental Toxicology and Pharmacology, vol. 49, pp. 188-193, 2017.

[13] Z. Lyv, T. Chen, L. Mao et al., "The chest CT imaging characteristics of mycoplasma pneumoniae with different age groups of children," Radiology of Infectious Diseases, vol. 4, no. 4, pp. 150-156, 2017. 
[14] N. Miyashita, T. Sugiu, Y. Kawai et al., "Radiographic features of Mycoplasma pneumoniae pneumonia: differential diagnosis and performance timing," BMC Medical Imaging, vol. 9, no. 1, pp. 7-8, 2009.

[15] P. Lomoro, F. Verde, F. Zerboni et al., "COVID-19 pneumonia manifestations at the admission on chest ultrasound, radiographs, and CT: single-center study and comprehensive radiologic literature review," European Journal of Radiology Open, vol. 7, Article ID 100231, 2020.

[16] A. Bartolomé, T. Pirogova, M. J. Bartolomé, R. Sánchez, and F. García de Lucas, "Glenohumeral instability: validity of lowfield MRI for diagnosis of labral tears," The Egyptian Journal of Radiology and Nuclear Medicine, vol. 47, no. 4, pp. 1521-1528, 2016.

[17] M. Baque-Juston, A. Pellegrin, S. Leroy, C. H. Marquette, and B. Padovani, "Organizing pneumonia: what is it? a conceptual approach and pictorial review," Diagnostic and Interventional Imaging, vol. 95, no. 9, pp. 771-777, 2014.

[18] J. H. Baik, J. M. Ko, and H. J. Park, "Pitfalls in radiographic interpretation of emphysema patients," Canadian Association of Radiologists Journal, vol. 67, no. 3, pp. 277-283, 2016.

[19] S. D. John, J. Ramanathan, and L. E. Swischuk, "Spectrum of clinical and radiographic findings in pediatric mycoplasma pneumonia," Radiographics, vol. 21, no. 1, pp. 121-131, 2001.

[20] M. Abdelsalam, H. S. Diab, and Y. Ragab, "Radiological findings in patients with H1N1 influenza pneumonia," Egyptian Journal of Chest Diseases and Tuberculosis, vol. 65, no. 1, pp. 135-142, 2016.

[21] M. Ohana, M. Y. Jeung, A. Labani, S. El Ghannudi, and C. Roy, "Thoracic dual energy CT: acquisition protocols, current applications and future developments," Diagnostic and Interventional Imaging, vol. 95, no. 11, pp. 1017-1026, 2014.

[22] Z. Luo, J. Luo, E. Liu et al., "Effects of prednisolone on refractory mycoplasma pneumoniae pneumonia in children," Pediatric Pulmonology, vol. 49, no. 4, pp. 377-380, 2014.

[23] B. Medjo, M. Atanaskovic-Markovic, S. Radic, D. Nikolic, M. Lukac, and S. Djukic, "Mycoplasma pneumoniae as a causative agent of community-acquired pneumonia in children: clinical features and laboratory diagnosis," Italian Journal of Pediatrics, vol. 40, no. 1, pp. 104-107, 2014.

[24] D. Kumar, A. Sharma, R. Kumar, and N. Sharma, "Restoration of the network for next generation $(5 \mathrm{G})$ optical communication network," in Proceedings of the 2019 International Conference on Signal Processing and Communication (ICSC), pp. 64-68, Noida, India, March 2019.

[25] M. Poongodi, A. Sharma, V. Vijayakumar et al., "Prediction of the price of Ethereum blockchain cryptocurrency in an industrial finance system," Computers \& Electrical Engineering, vol. 81, 2020.

[26] G. H. Mccracken Jr., "Diagnosis and management of pneumonia in children," The Pediatric Infectious Disease Journal, vol. 19, no. 9, pp. 924-928.

[27] T. Q. Tan, E. O. Mason, E. R. Wald et al., "Clinical characteristics of children with complicated pneumonia caused by Streptococcus pneumoniae," Pediatrics, vol. 110, no. 1, pp. 1-6, 2002.

[28] B. Lassmann, M. Poetschke, P. Apfalter et al., "Communityacquired pneumonia in children in Lambarene, Gabon," The American Journal of Tropical Medicine and Hygiene, vol. 79, no. 1, pp. 109-114, 2008.

[29] A. B. Cengiz, G. Kanra, M. Caĝlar, A. Kara, S. Güçer, and T. Ince, "Fatal necrotizing pneumonia caused by group A streptococcus," Journal of Paediatrics and Child Health, vol. 40, no. 1, pp. 69-71, 2004.
[30] M. P. Muller, D. E. Low, K. A. Green et al., "Clinical and epidemiologic features of group a streptococcal pneumonia in Ontario, Canada," Archives of Internal Medicine, vol. 163, no. 4, pp. 467-472, 2003.

[31] O. Wisesa, A. Adriansyah, and O. I. Khalaf, "Prediction analysis sales for corporate services telecommunications company using Gradient boost algorithm," in Proceedings of the 2nd International Conference on Broadband Communications, Wireless Sensors and Powering (BCWSP), pp. 101-106, Yogyakarta, Indonesia, September 2020.

[32] A. F. Subahi, Y. Alotaibi, O. I. Khalaf, and F. Ajesh, "Packet drop battling mechanism for energy aware detection in wireless networks," Computers, Materials and Continua, vol. 66, no. 2, pp. 2077-2086, 2020.

[33] X. Xiang, Q. Li, S. Khan, and O. I. Khalaf, "Urban water resource management for sustainable environment planning using artificial intelligence techniques," Environmental Impact Assessment Review, vol. 86, Article ID 106515, 2021.

[34] J. L. Basiliere, H. W. Bistrong, and W. F. Spence, "Streptococcal pneumonia," The American Journal of Medicine, vol. 44, no. 4, pp. 580-589, 1968.

[35] K. Mleczko-Sanecka, G. Casanovas, A. Ragab et al., "SMAD7 controls iron metabolism as a potent inhibitor of hepcidin expression," Blood, vol. 115, no. 13, pp. 2657-2665, 2010.

[36] P.-P. Cheng, Z.-Z. Sun, F. Jiang, Y.-T. Tang, and X.-Y. Jiao, "Hepcidin expression in patients with acute leukaemia," European Journal of Clinical Investigation, vol. 42, no. 5, pp. 517-525, 2012.

[37] A. C. Nascimento-Carvalho, O. Ruuskanen, and C. M. Nascimento-Carvalho, "Comparison of the frequency of bacterial and viral infections among children with community-acquired pneumonia hospitalized across distinct severity categories: a prospective cross-sectional study," $B M C$ Pediatrics, vol. 16, no. 1, p. 105, 2016.

[38] M. Krichen, S. Mechti, R. Alroobaea et al., "A formal testing model for operating room control system using internet of things," Computers, Materials \& Continua, vol. 66, no. 3, pp. 2997-3011, 2021.

[39] O. I. Khalaf, K. A. Ogudo, and M. Singh, "A fuzzy-based optimization technique for the energy and spectrum efficiencies trade-off in cognitive radio-enabled 5G network," Symmetry, vol. 13, no. 1, p. 47, 2021.

[40] O. I. Khalaf, F. Ajesh, A. A. Hamad, G. N. Nguyen, and D.-N. Le, "Efficient dual-cooperative bait detection scheme for collaborative attackers on mobile ad-hoc networks," IEEE Access, vol. 8, pp. 227962-227969, 2020.

[41] A. A. Hamad, A. S. Al-Obeidi, E. H. Al-Taiy, O. Ibrahim Khalaf, and D.-N. Le, "Synchronization phenomena investigation of a new nonlinear dynamical system 4-D by Gardano's and Lyapunov's methods," Computers, Materials \& Continua, vol. 66, no. 3, pp. 3311-3327, 2021.

[42] O. I. Khalaf and G. M. Abdulsahib, "Energy efficient routing and reliable data transmission protocol in WSN," International Journal of Advances in Soft Computing and its Application, vol. 12, pp. 345-353, 2020.

[43] O. I. Khalaf, G. M. Abdulsahib, and B. M. Sabbar, "Optimization of wireless sensor network coverage using the Bee algorithm," Journal of Information Science and Engineering, vol. 36, no. 2, pp. 377-386, 2020.

[44] S. K. Prasad, J. Rachna, O. I. Khalaf, and D.-N. Le, "Map matching algorithm: real time location tracking for smart security application," Telecommunications and Radio Engineering, vol. 79, no. 13, pp. 1189-1203, 2020.

[45] S. A. Rhedin, A. Eklundh, M. Ryd-Rinder et al., "Introducing a new algorithm for classification of etiology in studies on 
pediatric pneumonia: protocol for the trial of respiratory infections in children for enhanced diagnostics study," JMIR Research Protocols, vol. 8, no. 4, Article ID e12705, 2019.

[46] H. N. Njuguna, S. R. Zaki, D. J. Roberts et al., "Determining the cause of death among children hospitalized with respiratory illness in Kenya: protocol for pediatric respiratory etiology surveillance study (PRESS)," JMIR Research Protocols, vol. 8, no. 1, Article ID e10854, 2019. 